\title{
Combined Capillary Gas Chromatography/Ion Trap Mass Spectrometry Quantitative Methods Using Labeled or Unlabeled Internal Standards
}

\author{
R. J. Strife, J. R. Simms, and M. P. Lacey \\ Corporate Research Division, Miami Valley Laboratories, The Procter \& Gamble Company, \\ Cincinnati, Ohio, USA
}

\begin{abstract}
Three methods of operating an ion trap mass spectrometer (ITMS) were investigated for the determination of quantitative information by combined capillary GC/MS and GC/MS/MS. Separate regression lines for a hexynone drug were examined by using both unlabeled and stable-isotope-labeled internal standards. The ITMS modes of operation examined were (1) the full-scan, rf-voltage-only mode, useful on ion trap detectors as well as on the ITMS, (2) a scan using combined $\mathrm{rf}$ and dc voltages ( $\mathrm{rf} / \mathrm{dc}$ ) for mass-selective storage analyses, and (3) rf/dc followed by collision-induced dissociation. Results of over 200 analyses in the 0.1-10 ng on-column range, with $5 \mathrm{ng}$ of internal standard, showed that the unlabeled internal standard, separated by retention time on the capillary GC column, gave the best relative standard deviations (less than $5 \%$ over the range) and linear correlations ( $r^{2}$ typically > 0.9992). (J Am Soc Mass Spectrom 1990, 1, 265-271)
\end{abstract}

$\mathrm{T}$ The ion trap mass spectrometer (ITMS) is an ion storage device [1-3]. An exploded view and cross section of the trapping structure were published earlier [3]. The device has cylindrical symmetry $(r, z$ geometric coordinates), with an electrically isolated ring electrode of $1 \mathrm{~cm}$ radius centered on the $z$ axis. The surface of the ring electrode is hyperbolic, and the trap structure has grounded hyperbolic end caps. By application of $\mathrm{r} f$ and/or dc voltages to the ring electrode, a rotationally symmetric quadrupole electric field is established. Ions formed in this field can be trapped in stable trajectories.

The various stages of a mass spectrometry experiment are carried out one step at a time, in the presence of He buffer gas $\left(10^{-4}\right.$ torr), with each step taking typically $0.1-25 \mathrm{~ms}$, depending on the purpose. For instance, as neutral molecules stream into the trap, a simple electron ionization (EI) mass spectral analysis is carried out by (1) applying a relatively low, fixed if voltage to the ring electrode, (2) creating the family of ions making up the mass spectrum of using a pulse of electrons (0.1-25 ms depending on the sample concentration), (3) allowing the formed ions to damp down into stable orbits near the center of the trap $(1 \mathrm{~ms})_{\text {r }}(4)$ turning on the external electron multiplier (1 ms), and (5) ramping the of voltage upward. In this last step, ions of sequentially greater $\mathrm{m} / \mathrm{z}$ value become unsta-

Address reprint requests to Dr. Robert J. Strife, Corporate Research Division, Miami Valley Laboratories, Procter \& Gamble Company, P.O. Box 398707, Cincinnati, OH 45239-8707. ble in the $z$ component of their motion and leave the trap along the $z$ axis, striking the detector. This scan of the mass spectrum, referred to as the rf-only mode of operation, is done at $18 \mathrm{~ms} / 100 \mathrm{u}$. Accounting for real-time evaluations carried on by the data system (automatic gain control, or AGC, described within), a fullscan experiment is completed in less than $150 \mathrm{~ms}$ [4]. Spectra of good quality are obtained by GC/MS at the 50-pg level or lower by electron or chemical ionization of analytes [5-7].

Alternatively, the initial population of stored ions may be manipulated by using rapid rf ramps (1-2 ms) and a dc voltage pulse (designated the rf/dc method) to cause storage of ions of only a particular $m / z$ value. All other ions go into unstable trajectories and leave the trap. If the detector is then turned on and the remaining stored ions are scanned out as before, the collected data are analogous to those from a selected-ion monitoring experiment on a "standard" quadrupole. (Standard, as used here, refers to a quadrupole mass spectrometer using a rectangular array of rods to establish the field.) The steps of the rf/dc scan function have been described in detail elsewhere in relation to the theoretical stability diagram for ion trajectories in the trap [8]. The process is called a mass-selective storage analysis.

This rf/dc approach has advantages for target compound analyses. Relatively long ( $25 \mathrm{~ms}$ ) ionization pulses can be used, resulting in an overfilled (spacecharged) trap. Spectral resolution temporarily decreases as the mass peaks broaden and shift in their 
assigned $m / z$ values. These effects are due to the ions being subject to each other's electric fields as well as that from the ring electrode. Then the $\mathrm{rf} / \mathrm{dc}$ sequence ejects all ions except those of the desired $m / z$ value, decreasing the total ion population and correcting the space-charge condition [9]. Enhanced response is obtained for the analyte because many more ions are created initially by the long ionization pulse.

A third alternative is to carry out collision-induced dissociation (CID) MS/MS of the ions isolated by rf/dc. This is accomplished by applying an ac voltage pulse (typically a few hundred millivolts) across the end caps of the trap for about $20 \mathrm{~ms}$ after the ions of interest are isolated by rf/dc. The frequency of the voltage is resonant with the fundamental frequency of oscillation of the ions along the $z$ axis. As the kinetic energy of the ions increases and the magnitude of their excursions in the $z$ direction increases, they undergo collisions with the He buffer gas. The rf level for this part of the experiment is set to allow trapping of the product ions as they form. The mass analysis is carried out as before, by turning on the detector and ramping the rf voltage.

Greater selectivity would be expected in the analysis of biological extracts, by detecting product ions of the stored parent ions. More important, CID has high observed efficiency, typically $50-100 \%$, and this results in high-sensitivity MS/MS analyses [3, 6].

Even more complex tandem experiments (e.g., sequential daughter MS/MS/MS) can be carried out on the ITMS, but for analytical utility the three modes described above are most common, representing (1) fullscan, rf-only mass spectral experiments; (2) enhancedsensitivity target compound analysis using $\mathrm{rf} / \mathrm{dc}$ to cause mass-selective storage; and (3) enhanced selectivity due to $\mathrm{rf} / \mathrm{dc}$ isolation of parent ions followed by MS/MS. We had applied the latter approach to the qualitative GC/MS/MS analysis of tryptamine in a brain tissue extract [6] and needed to define the quantitative performance of the ITMS more precisely in further biological applications.

Capillary gas chromatography (GC) is a common approach to sample introduction in ion trap experiments where low detection limits (picogram level) are desired. Qualitative work has been reported by many authors, but little information exists on quantitative analyses on the ITMS when internal standards (IS) are used. We therefore selected a stable and pure compound, NE-11740, formally 1-[3,5-bis(1,1-dimethylethyl)-4-hydroxyphenyl]5-hexyn-1-one, which is an anti-inflammatory drug in clinical trials. Two types of IS were available. They were a ${ }^{13} \mathrm{C},{ }^{18} \mathrm{O}$ stable-isotope-labeled analogue (stable-isotope standard, or SIS), which coeluted with NE-11740, and an unlabeled chemical structure variant (chemical internal standard, or CIS), which separated by retention time. Quantitative GCiMS and GC/MS/MS analyses of these compounds on "standard" quadrupole instruments had shown good linearity and dynamic range. The $r^{2}$ value was $>0.9995$ over three orders of magnitude [10]. This base of data was deemed very useful for evaluating the ion trap. We report herein the results of regression analyses using the pure standards.

\section{Experimental}

\section{Materials and Methods}

The three compounds used in this study were synthesized, purified, and fully characterized at Procter \& Gamble's Miami Valley Laboratories. They included the hexynones NE-11740, a 3-methyl analogue as the CIS, and ${ }^{13} \mathrm{C},{ }^{18} \mathrm{O}-\mathrm{NE}-11740$ (both labels at the 1 position of the hexynone moiety) as the SIS. All solvents used were of HPLC grade. The three compounds were weighed accurately $( \pm 0.05 \mathrm{mg})$ into separate $10-\mathrm{mL}$ volumetric flasks, to a value near $50 \mathrm{mg}$, using a Mettler electronic balance. Serial dilutions were carried out in acetone. Two standard curves were made for NE11740 by mixing and accurately diluting the stock solutions described above. These new solutions were made up in pairs. Aliquots of NE-11740 were dispensed to volumetric flasks containing the unlabeled CIS or the SIS. The final solutions analyzed ranged in concentration from about $10 \mu \mathrm{g} / \mathrm{mL}$ to $1 \mathrm{mg} / \mathrm{mL}$ NE-11740. In all cases, the IS concentration was close to $0.5 \mathrm{mg} / \mathrm{mL}$. Thus, the final weight ratios ranged from 0.02 to 2 , covering two orders of magnitude.

\section{Instrumentation}

Gas chromatography was carried out on a $15 \mathrm{~m} \times 0.25$ mm i.d. DB-1 or DB-5 column directly interfaced to the mass spectrometer. The film thickness was always $0.25 \mu \mathrm{m}$. Injections were done in the split mode with a measured split ratio of $(100 \pm 5) / 1$ on either GCMS system. This resulted in $0.1-10 \mathrm{ng}$ of NE-11740 and 5 ng of IS entering the system (Table 1). The injector and transfer line temperatures were $250^{\circ} \mathrm{C}$. The column

Table 1. Summary of calibration sample amounts on column and ionization time ${ }^{a}$

\begin{tabular}{|c|c|c|c|c|c|c|c|}
\hline $\begin{array}{l}\text { Amount NE- } 11740 \\
\text { on column (ng) }\end{array}$ & 0.100 & 0.251 & 0.502 & 1.00 & 2.51 & 5.02 & 10.04 \\
\hline $\begin{array}{l}\text { Ionization time }(\mu \mathrm{s}) \\
\quad \text { for non- } \mathrm{AGC} \text { expe }\end{array}$ & 10.000 & 4,000 & 2,000 & 1,000 & 400 & 200 & 100 \\
\hline
\end{tabular}

a Based on known concentrations (1 $\mu \mathrm{L}$ injected) and 100:1 split ratio. The CIS amount was $5.36 \mathrm{ng}(200 \mu \mathrm{s}$ ionization time), and the SIS amount was $5.07 \mathrm{ng}$ (200 $\mu$ s ionization time). 
was programmed from $130^{\circ} \mathrm{C}(1 \mathrm{~min})$ to $250^{\circ} \mathrm{C}$ at 10 ${ }^{\circ} \mathrm{C} / \mathrm{min}$.

Reference calibration lines were obtained by GC/MS using a Hewlett-Packard 5985B system, a "standard" quadrupole system, in the selected-ion-monitoring mode (electron ionization, $70 \mathrm{eV}, 200 \mu \mathrm{A}$, and $50 \mathrm{~ms}$ dwell time). The prototype ITMS has been described [3], and design changes are noted in the manufacturer's literature (Finnigan-MAT, San Jose, CA). A modified 6-in. conflat flange was constructed to accept a TSQ-70 GC/MS transfer line (Finnigan-MAT). The transfer line was interfaced to an HP 5890 GC according to the manufacturer's guidelines.

The ITMS was controlled by software release 4.1 ITD and version 1.41 scan-editor software with a Forth program screen editor. Ionization rf levels were set at the equivalent of $20 \mathrm{u}$ in the software parameters, and the filament current was $20 \mu \mathrm{A}$. In the rf-only, full-scan experiments, automatic gain control (AGC) software was used. Under AGC control, a shortened ionization/scan sequence is used to determine the relative concentration of all materials present in the trap. The ionization pulse is set by the data system to maximally fill the trap with ions. For the other experiments (rf/dc and $\mathrm{rf} / \mathrm{dc}$ MS/MS), this was not an option, and the ionization pulse was fixed, although it could be changed to a different fixed value during a run. For the optimization of table parameters controlling the ITMS, samples were boiled slowly off the direct-inlet probe. Mouse-driven software was used to optimize mass-selective storage $\mathrm{rf} / \mathrm{dc}$ and MS/MS parameters in real time. Alternating scan functions were run by utilizing the Forth program screen editor of the software. The scan functions for these experiments have been previously described [6, 8, 9].

\section{Results and Discussion}

\section{Gas Chromatography}

The structures of NE-11740, the 3-methyl analogue used as the CIS, and positions for the labels in the SIS of NE-11740 are shown in Figure 1. The GC/MS analysis (rf-only) of a mixture of NE-11740 and the CIS is shown in Figure 2. The retention time of NE-11740 was about $5.5 \mathrm{~min}$ on the ITMS system, and the CIS

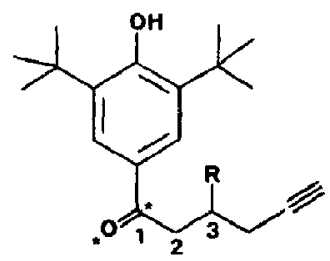

Figure 1. Structures of NE-11740 ( $R=H$ ), the chemical internal standard (CIS) $\left(\mathrm{R}=\mathrm{CH}_{3}\right)$, and the stable-isotope-labeled internal standard (SIS, $R=H$, position of ${ }^{18} \mathrm{O}$ and ${ }^{13} \mathrm{C}$ indicated by ${ }^{*}$ symbol).

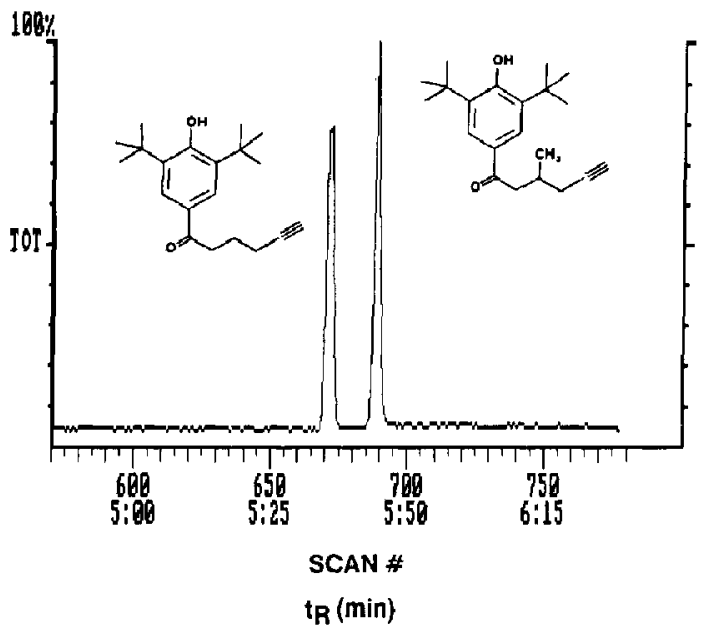

Figure 2. Combined capillary GC/MS analysis of NE-11740 $\left(t_{R}=\right.$ 5:35) and the 3-methyl analogue $\left(t_{R}=5: 45\right)$. TOT $=$ total ion current.

eluted $10 \mathrm{~s}$ later. The SIS coeluted with NE-11740 as was expected. The $k^{\prime}$ value is about 10 , with a peak width at half-height of $2 \mathrm{~s}$. The GC profiles were very symmetric on both GC/MS systems. Injection of relatively concentrated samples $(0.5 \mathrm{mg} / \mathrm{mL}$ for the IS) at a high split ratio was chosen, because we desired to establish the performance of the mass spectrometer. In our laboratory this type of injection has been the least problematic in GC operation and presents the least disturbance of the ITMS system.

\section{Mass Spectral Characterization of Standards}

The mass spectrum of NE-11740 in Figure 3 is from the ITMS. The initial ionic structures shown are derived from classical rules of fragmentation. The interpretation of the spectrum is straightforward. A molecular ion peak is visible at $m / z$ 300. Cleavage by McLafferty rearrangement produces the radical cation of $\mathrm{m} / \mathrm{z}$ 248 (base peak), eliminating the comparatively stable 1-buten-3-yne. The ion at $m / z 233$ arises from $\alpha$ cleavage of the ketone, to produce the even-electron ion. Studies by MS/MS, using the ITMS, show that the $m / z$ 248 species loses a methyl radical to also form an ion of $m / z 233$. This can occur by two possible pathways: (1) enolization of the McLafferty product to the methyl phenone form, followed by $\alpha$ cleavage, or (2) cleavage of a methyl radical from the tert-butyl group. Other experiments using a 2,2-deuterated NE-11740 show that cleavage of methyl from the tert-butyl group occurs about $25 \%$ of the time, because the deuterium is retained [11]. This is not expected for exclusive $\alpha$ cleavage. The fragmentations are also confirmed by the 3-u shifts observed for the $m / z 233-236$ and $m / z$ 248-251 peaks for ${ }^{13} \mathrm{C},{ }^{18} \mathrm{O}$-labeled NE-11740. The isotopic distribution by relative abundances was $m / z 248(0.4 \%)$, $m / z 249(7.2 \%), m / z 250(5.6 \%), m / z 251$ (100\%). This 
Figure 3. Electron ionization mass spectrum for NE-11740. Initial ion structures, based on classical fragmentation rules, are shown for $m / z 248$ and $m / z 233$.

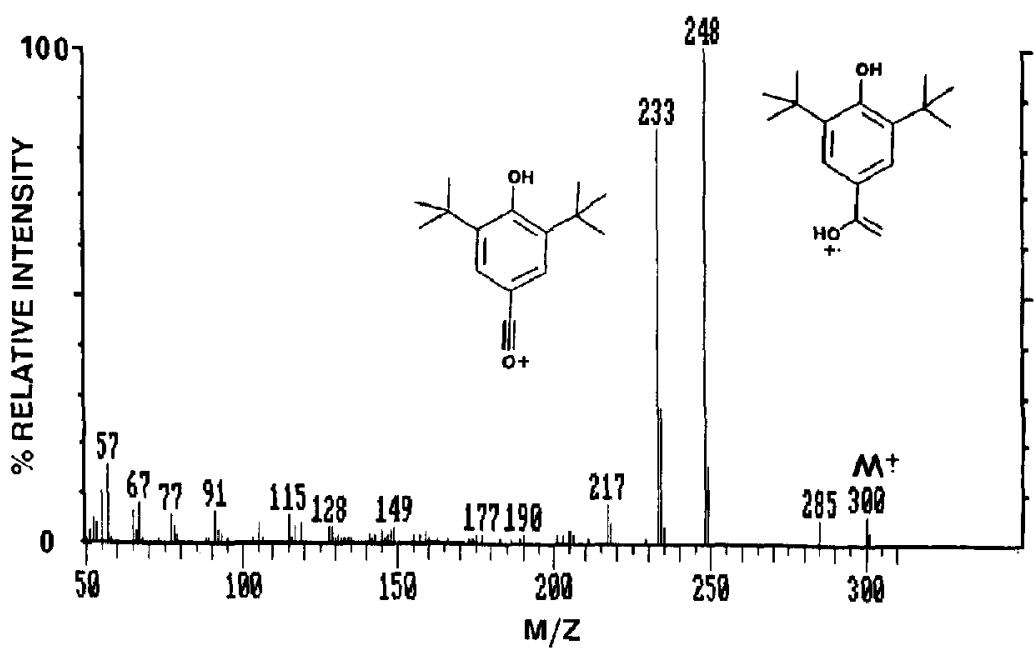

concentration solution (100 pg of analyte with $5 \mathrm{ng}$ of IS) was analyzed with $n=8$. For the CIS curve, a plot of the response ratio for NE-11740 to that of the 3-methyl analogue (using $\mathrm{m} / \mathrm{z} 248$ ) versus their weight ratio showed

$$
y=0.769 x-0.005 \quad \text { with } r^{2}=0.9999
$$

The slope reflects the fact that the IS has more hydrogen atoms available for McLafferty rearrangement than does NE-11740. The CIS therefore has a higher response factor for $m / z 248$ on a mole basis, and the observed slope is less than 1 . At the low concentration point (100 pg of NE-11740 on column), the relative standard deviation (RSD) was $2.7 \%(n=8)$.

For the SIS curve, a standard plot of the response ratio of $m / z 248$ to $m / z 251$ versus the weight ratio of NE-11740 to labeled NE-11740 gave

$$
y=1.221 x-0.001 \quad \text { with } r^{2}=0.9999
$$

The RSD at the low point was $1.1 \%$. The slope for the stable isotope line reflects the fact that the IS of

Table 2. Summary of regression line analyses ${ }^{a}$

\begin{tabular}{lcccccc}
\hline Method & $\begin{array}{c}\text { Internal } \\
\text { standard }\end{array}$ & Regression line & $r^{2}$ & RSD (\%) & $n$ \\
\hline Standard quad. & $\mathrm{C}$ & $y=0.769 x-0.005$ & 0.9999 & 2.7 & 8 \\
Standard quad. & $\mathrm{S}$ & $y=1.22 x-0.001$ & 0.9999 & 1.1 & 8 \\
ITMS-AGC rf-only & $\mathrm{C}$ & $y=0.805 x-0.008$ & 0.9992 & 6.0 & 5 \\
ITMS-AGC rf-only & $\mathrm{S}$ & $y=1.151 x-0.051$ & 0.9927 & 20.0 & 5 \\
ITMS rf/dc & $\mathrm{C}$ & $y=0.857 x+0.011$ & 0.9998 & 3.0 & 8 \\
ITMS rf/dc & $\mathrm{S}$ & $y=0.907 x-0.006$ & 0.9993 & $-{ }^{0}$ & - \\
ITMS rf/dc & $\mathrm{S}$ & $y=1.264 x-0.000$ & 0.9901 & 50.0 & 8 \\
ITMS rf/dc MS/MS & $\mathrm{C}$ & $y=0.854 x+0.011$ & 0.9997 & 3.7 & 8 \\
\hline
\end{tabular}

"Quad. = quadrupole; internal standard is chemical (C) or stable isotope (S).

- Single points of 20 averaged scans from direct inlet probe were plotted. The scan-to-scan reproducibility of the isotope ratio was $5 \%$ at the low concentration. 
$\mathrm{NE}-11740$ is not $100 \%{ }^{13} \mathrm{C},{ }^{18} \mathrm{O}$-labeled. The slope is therefore greater than 1 . Both methods show excellent linearity, $y$ intercepts, and reproducibility. With the integrity of the dilution curves verified by this standard method, we proceed to analyze the same solutions on the ITMS.

\section{Full-Scan, RF-Only ITMS Analyses Under AGC Control}

An attractive feature of ion traps is the sensitivity observed for full-scan GC/MS analyses. Selected-ion monitoring traces are simply reconstructed after the run.

The CIS Curve. These samples were run at $0.5 \mathrm{~s}$ per scan (the fastest scan speed allowed by the software), with three scans (called microscans) averaged at each of these $0.5-\mathrm{s}$ intervals. Postrun processing was used to reconstruct the ion chromatogram for the $m / z 248$, and the areas were integrated by the data system. Each sample was run five times. The line obtained was

$$
y=0.805 x-0.008 \quad \text { with } r^{2}=0.9992
$$

The RSD averaged about $6 \%$ at each point, and the linearity and $y$ intercept were quite acceptable. The rate of $0.5 \mathrm{~s}$ per scan limited the definition of the capillary GC peak to six data points, whereas data were integrated every $50 \mathrm{~ms}$ on the standard quadrupole. This may account for observed statistical differences. Nevertheless, it is our opinion that this approach is quite feasible where the analyte/matrix ratio favors the analyte. The slight decrease in reproducibility is offset by the fact that full-scan spectral information is always obtained when the ion trap is used.

The SIS Curve. We expected the stable-isotope dilution curve to be more problematic in the rf-only mode, because the SIS coelutes with the analyte. Automatic gain control software adjusts the ionization time downward according to the total concentration of all components. This creates a paradox of sorts. When the analyte concentration is low (100 pg of NE-11740), a long ionization time $(10 \mathrm{~ms})$ is desired. However, the coeluting internal standard ( $5 \mathrm{ng}$ ) causes a low ionization time $(75 \mu \mathrm{s})$ to be selected, resulting in poor signal for the $m / z 248$ trace characteristic of the analyte. Even if a long fixed ionization time is used, the high level of IS causes significant space charge to develop and gives an unsatisfactory result in the rf-only mode.

The line obtained by postrun processing was

$$
y=1.151 x-0.051 \quad \text { with } r^{2}=0.9927
$$

However, the average RSD was $20 \%$. We suspect that ion statistics play a role here. It may be that enough ions are not being created for the analyte to be repro- ducibly analyzed and that this leads to scatter in the ratios obtained. z-Axial modulation [9] allows a tenfold increase in the total number of ions stored in the trap, so it may be beneficial in this case. We are currently testing this hypothesis. We are also examining narrower dynamic ranges where analyte and IS concentrations are more closely matched. It appears that internal standards separated by retention time may be superior for full-scan, rf-only analyses. Automatic gain control can then optimize the ionization time for each component. However, more systems must be examined before a general conclusion is reached. Also, applications to biological extracts should be carefully evaluated, because coeluting matrix materials may also decrease ionization times selected by AGC. Adequate sample cleanup will be important.

\section{RF/DC Mass-Selective Storage Analyses}

We have previously given the description of the $\mathrm{rf} / \mathrm{dc}$ scan function used in conjunction with the massselective instability scan mode [8]. This process causes a mass-selective-storage condition. These ions may be scanned out or subjected to further manipulations like CID (MS/MS analysis) or gas-phase reactions [2, 12, 13] before the mass analysis step. At lower masses (less than $50 \mathrm{u}$ ), a single $m / z$ value may be isolated, but, in our experience, at higher masses (greater than 200 u) the ions at values within $1 \mathrm{u}$ of the selected $m / z$ value are not totally eliminated without a significant loss in signal of the desired ion. For instance, in isolating the $m / z 248$ ion, the $m / z 249$ ion is also trapped, but resolution of $m / z 248$ and 249 is maintained during mass analysis.

The CIS Curve. For analysis of these samples, a single scan function was used. NE-11740 and the CIS separate chromatographically and are both characterized by $m / z 248$, the base peak in their spectra (Figure 3). Therefore, only one mass-selective storage scan function was required. The ionization time used was a fixed value (i.e., not controlled by AGC) but could be changed during a GCIMS run to a new fixed value by using an interactive Forth program in the scan editor software (Table 1). The internal standard (5 $\mathrm{ng}$ ) was ionized for $200 \mu \mathrm{s}$, and the analyte was ionized for an inversely proportional time based on its concentration in the solution. For instance, $100 \mathrm{pg}$ of NE-11740 was ionized for $10 \mathrm{~ms}$. Thus, for any given data point on the curve, the numbers of ions created for NE-11740 and the CIS were about the same. The observed ratios of GC peak areas were corrected postrun for the ionization time factors. A typical result is shown in Figure 4, where nearly equal peak areas are observed for 250 pg of NE-11740 and $5 \mathrm{ng}$ of the CIS.

The equation found by regression analysis was

$$
y=0.935 x-0.0147 \quad \text { with } r^{2}=0.9991
$$




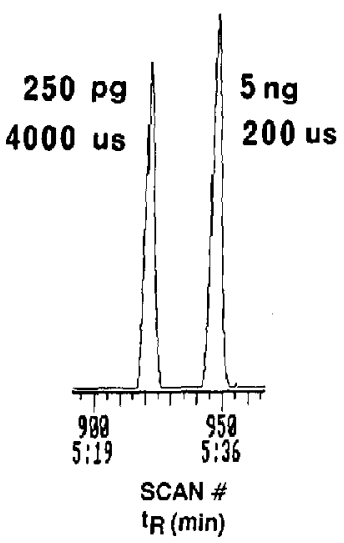

Figure 4. Combined capillary GCIMS analysis ( $\mathrm{rf} / \mathrm{dc}$, isolating $m / z 248$ ) of a low concentration point from the calibration line for the chemical internal standard. The $250 \mathrm{pg}$ of NE-11740 and 5000 pg of AI-098 produce nearly equal peak areas owing to selection of different ionization times (shown in $\mu \mathrm{s}$ ).

when the reconstructed trace for just $m / z 248$ was analyzed. A better result was obtained by analyzing the total ion trace, which included the natural abundance isotope peak at $m / z 249$. The equation was

$$
y=0.857 x+0.011 \quad \text { with } r^{2}=0.9998
$$

The RSD value was 3\% at the low-concentration point.

The SIS Curve. As pointed out in the rf-only AGC work, the SIS coelutes with NE-11740, creating a problem in selection of the ionization time. A long ionization time for $100 \mathrm{pg}$ of NE-11740 gives significant space charge for $5 \mathrm{ng}$ of the coeluting SIS, which is unsatisfactory for rf-only analyses [6]. Using the rf/dc method can help to obviate this problem because it can correct for the space charge. However, because the SIS coelutes, it becomes necessary to rapidly alternate back and forth between mass-selective storage scans for $m / z 248$ (NE-11740) and $m / z 251$ (SIS) so that an accurate representation of the capillary GC peak is obtained for both species. This is similar to "switching channels" back and forth in selected-ion monitoring on the "standard" quadrupole. The speed of switching scan functions back and forth is critical to defining the GC peak profile.

We therefore decided to first estimate the ion trap's capability to measure the isotope-dilution line under conditions where fast switching was not necessary. The SIS samples were boiled slowly off the direct-inlet probe to produce a relatively constant intensity while the alternating scan functions acquired the data. A reference line of $y=0.907 x-0.006$ was obtained with $r^{2}=0.9993$. The slope is noticeably lower than that obtained with the standard quadrupole, but good linearity is observed. We are not certain of the reason for this lower slope but suspect that differences in tuning the individual scan functions resulted in different storage efficiencies for the ions being analyzed.
When the same solutions were analyzed in the GC/MS mode with the alternating scan functions, the line obtained was

$$
y=1.264 x-0.005 \quad \text { with } r^{2}=0.9901
$$

The RSD at the lowest point, however, was $50 \%$. Even at the other points on the curve, the RSD values were found to be consistently $30-40 \%$. This is despite the fact that the number of ions formed for the analyte and the SIS is the same as in the CIS analysis, where RSD values of $3 \%$ were obtained. We therefore attribute the large RSDs at least partially to the slow effective scan acquisition rate in this alternating scan mode.

In the case of CIS, 10-15 scans/s were averaged for both of the analytes. Only one scan function was operative at any given point, because NE-11740 and the CIS separate by retention time. The number of scans obtained was dependent only on the ionization time. That is, when a long ionization time was chosen for a low level of NE-11740, the number of scans acquired decreased to $10 \mathrm{~s}^{-1}$. The additional time is consumed by the long ionization period.

In the case of the SIS, the alternating scan functions for $m / z 248$ and $m / z 251$ were each about $60-70$ ms long. It was found that these functions were accessed alternately from the hard disk drive and loaded into the trap firmware on a continuous basis. This is a relatively slow process. A total of two scans per second are acquired, resulting in poorer averaging or poorer GC peak shape definition, depending on the choice of the user. Fortunately, a change in the software should make possible faster accessing of these functions in an alternate fashion. We expect that RSDs of less than $5 \%$ will be attainable in the future with the SIS. Because a poor result was obtained in the mass-selective storage mode, we did not pursue GCMS/MS with the SIS.

\section{RF/DC with Subsequent MS/MS}

Having established a very good result with the CIS, we next examined quantitative GC/MS/MS. The scan function for GCMS/MS applications has been previously described [8]. The parent ion at $m / z 248$ was found to lose methyl radical exclusively to form an ion of $m / z 233$, with $100 \%$ apparent CID efficiency, when the ITMS was used. The fact that the daughter ion current appears in only one $m / z$ value with $100 \%$ efficiency should mean that this is a best-case example for GC/MS/MS on the ITMS. When the CIS-curve solutions were analyzed, the line

$$
y=0.854 x+0.011 \quad \text { with } r^{2}=0.9997
$$

was obtained, very comparable to standard quadrupole and other ITMS data. The RSD at the low concentration was $3.7 \%$. Thus, sensitive and accurate GCMS/MS may be possible on the ion trap. The outstanding feature of this approach is the extremely high observed CID efficiency. 
In summary, by choosing to run these evaluations with relatively concentrated solutions at a high split ratio, we have determined the optimal capabilities of the ITMS system. The mass spectrometer is not disturbed by large pressure fluctuations from the solvent of injection or matrix materials from biological samples. The largest obstacle to trace analysis is the coelution of other materials with the analyte, and the $\mathrm{rf} / \mathrm{dc}$ approach can abviate this problem. Faster switching speeds will have to be achieved for successful analyses based on coeluting internal standards. In the rfonly AGC case, stable-isotope-labeled internal standards that separate by retention time but behave virtually identically to the analyte during sample preparation [14] may be useful. We are now proceeding to evaluate performance of the system over three orders of magnitude and under more realistic conditions of biological sample analysis. These conditions are splitless or on-column injection of calibration samples derived from plasma extracts, using GC/MS/MS for optimized selectivity.

\section{References}

1. Dawson, P. H., Ed. Quadrupole Mass Spectrometry and Its Applications; Elsevier: New York, 1976.
2. March, R. E.; Hughes, R. J.; Todd, J. F. J. Quadrupole Storage Mass Spectrometry; Wiley: New York, 1989.

3. Louris, J. N.; Cooks, R. G.; Syka, J. E. P.; Kelly, P. E.; Stafford, G. C. Anal. Chem. 1987, 59, 1677-1685.

4. Stafford, G. C.; Taylor, D. M.; Bradshaw, S. C.; Syka, J. E. P.; Ulrich, M. Presented at the 35th ASMS Conference on Mass Spectrometry and Allied Topics, Denver, Col., May 24-29, 1987.

5. Yost, R. A.; McClennen, W.; Snyder, A. P. Presented at the 35th ASMS Conference on Mass Spectrometry and Allied Topics, Denver, Col, May 24-29, 1987.

6. Strife, R. J.; Simms, J. R. Anal. Chem. 1989, 61, 2316-2319.

7. Tucker, D. B.; Hameister, C. H.; Bradshaw, S. C.; Hoekaman, D. J.; Weber-Grabau, M. Presented at the 36th ASME Conference on Mass Spectrometry and Allied Topics, San Francisco, Cal., June 5-10, 1988.

8. Strife, R. J.; Kelley, P. E.; Weber-Grabau, M. Rapid Commun. Mass Spectrom. 1988, 2, 105-109.

9. Technical Report 908, Finnigan-MAT, San Jose, Cal., 1988.

10. Dobson, R. L.; Neal, D. M.; DeMark, B. R.; Ward, S. Presented at the 37th ASMS Conference on Mass Spectrometry and Allied Topics, Miami Beach, Fla., May 22-26, 1989.

11. Doyle, M. J.; Eichold, T. Biomed. Envirom. Mass Spectrom. 1990, in press.

12. Strife, R. J.; Keller, P. R. Org. Mass Spectrom. 1989, 24, 201-204.

13. Brodbelt, J. S.; Louris, J. N.; Cooks, R. G. Anal. Chem. 1984, 59, 1278-1285.

14. Keough, T.; Strife, R. J.; Rodriguez, P. A.; Sanders, R. A. J. Chromatogr. 1984, 312, 450-455. 